\title{
Surveillance of healthcare-associated infection in hospitalised South African children: Which method performs best?
}

\author{
A Dramowski, ${ }^{1} \mathrm{MB}$ ChB, DCH, FCPaed (SA), Cert Paed ID; M F Cotton, ${ }^{1} \mathrm{MB}$ ChB, DCH, FCPaed (SA), PhD; \\ A Whitelaw, ${ }^{2} \mathrm{MB} \mathrm{ChB}, \mathrm{MSc}$, FCPath (SA) (Micro) \\ ${ }^{1}$ Department of Paediatrics and Child Health, Division of Paediatric Infectious Diseases, Faculty of Medicine and Health Sciences, \\ Stellenbosch University, Cape Town, South Africa \\ ${ }^{2}$ Division of Medical Microbiology, Department of Pathology, Faculty of Medicine and Health Sciences, Stellenbosch University and \\ National Health Laboratory Service, Tygerberg Hospital, Cape Town, South Africa
}

Corresponding author: A Dramowski (dramowski@sun.ac.za)

\begin{abstract}
Background. In 2012, the South African (SA) National Department of Health mandated surveillance of healthcare-associated infection (HAI), but made no recommendations of appropriate surveillance methods.

Methods. Prospective clinical HAI surveillance (the reference method) was conducted at Tygerberg Children's Hospital, Cape Town, from 1 May to 31 October 2015. Performance of three surveillance methods (point prevalence surveys (PPSs), laboratory surveillance and tracking of antimicrobial prescriptions) was compared with the reference method using surveillance evaluation guidelines. Factors associated with failure to detect HAI were identified by logistic regression analysis.

Results. The reference method detected 417 HAIs among 1347 paediatric hospitalisations (HAI incidence of 31/1000 patient days; 95\% confidence interval (CI) 28.2 - 34.2). Surveillance methods had variable sensitivity (S) and positive predictive value (PPV): PPS S = 24.9\% (95\% CI 21 - 29.3), PPV = 100\%; laboratory surveillance S = 48.4\% (95\% CI $43.7-53.2)$, PPV = 55.2\% (95\% CI $50.1-60.2)$; and antimicrobial prescriptions $\mathrm{S}=66.4 \%$ (95\% CI $61.8-70.8 \%), \mathrm{PPV}=88.5 \%$ (95\% CI 84.5 - 91.6). Combined laboratory-antimicrobial surveillance achieved superior HAI detection ( $\mathrm{S}=84.7 \%(95 \% \mathrm{CI} 80.9-87.8 \%)$, PPV $=97 \%$ (95\% CI $94.6-98.4 \%)$ ). Factors associated with failure to detect HAI included patient transfer (odds ratio (OR) 2.0), single HAI event (OR 2.8), age category 1 - 5 years (OR 2.1) and hospitalisation in a general ward (OR 2.3).

Conclusions. Repeated PPSs, laboratory surveillance and/or antimicrobial prescription tracking are feasible HAI surveillance methods for low-resource settings. Combined laboratory-antimicrobial surveillance achieved the best sensitivity and PPV. SA paediatric healthcare facilities should individualise HAI surveillance, selecting a method suited to available resources and practice context.
\end{abstract}

S Afr Med J 2017;107(1):56-63. DOI:10.7196/SAMJ.2017.v107i1.11431

Healthcare-associated infections (HAIs) are the most common complication of hospitalisation, resulting in adverse patient outcomes and increased healthcare costs. ${ }^{[1]}$ The burden of HAIs in most high-income settings is well established by national internet-based reporting systems or repeated national/facility-level HAI point prevalence surveys (PPSs). ${ }^{[2-5]}$

In sub-Saharan Africa, most healthcare facilities are unable to perform HAI surveillance because they lack trained infection prevention (IP) staff, data analysts and information technology (IT) infrastructure ${ }^{[6]}$ The situation in South Africa (SA), a country with greater resources than many of its neighbours, is similar, with no national HAI surveillance programme and extremely limited data on the paediatric and adult HAI burden. ${ }^{[7,8]}$ A single study in 2005 estimated a prevalence of $9.7 \%$ for four major HAI types, with higher prevalence among children (16.5\%) and patients in intensive care units (ICUs) (28.5\%). ${ }^{[9,10]}$

In 2012, SA introduced National Core Standards for Healthcare Establishments, ${ }^{[1]}$ with a patient safety domain mandating HAI surveillance, but lacking recommendations for HAI surveillance methods. Prospective clinical (patient-based) surveillance is considered the reference method or 'gold standard' for HAI surveillance, but requires substantial resources. ${ }^{[12,13]}$ HAI surveillance is conventionally conducted by IP staff applying technically complex definitions to their inpatient population (most often using the Centers for Disease Control's National Healthcare Safety Network (CDC/NHSN) definitions ${ }^{[14]}$. Even in well-resourced settings, so-called 'whole-house' clinical surveillance for all HAI event types is seldom done. Instead, targeted HAI surveillance is more commonly performed, focusing on specific clinical units (e.g. ICUs), types of HAI (e.g. device-associated infection) or procedures (e.g. surgical site infection (SSI) after caesarean section). Less resource-intensive HAI surveillance methods that still allow for monitoring of HAI trends and benchmarking are required, especially in resource-limited settings. ${ }^{[3,5]}$

Electronic detection of HAIs has many advantages over conventional clinical surveillance: patient data abstraction is automated, and several data sources can be combined. This method increases objectivity, reliability and efficiency of HAI identification. ${ }^{[15]}$ However, automated surveillance may be a poor proxy for conventional HAI surveillance (particularly when utilising the 10th revision of the International Statistical Classification of Diseases and Related Health Problems (ICD-10) coding), with both false positives and missed HAI events reported. ${ }^{[16-18]}$ Computerised HAI identification algorithms that use multiple sources of information (e.g. laboratory data, ICD-10 coding and inpatient prescriptions) can achieve better sensitivity and positive predictive values (PPVs). ${ }^{[19]}$ 
However, lack of IT infrastructure and electronic health records precludes the use of automated HAI surveillance in most low- and middle-income countries (LMICs), including SA.

Data on device-associated infection rates in the private SA healthcare sector were recently published ${ }^{[20]}$ highlighting the challenges associated with active HAI surveillance even in a comparatively well-funded setting. Alternative, passive HAI surveillance methods that could be utilised in low-resource settings include laboratory surveillance (for selected hospital-acquired pathogens) and review of pharmacy prescriptions (identifying antimicrobials used to treat HAIs). In addition, repeated PPSs may be feasible in LMIC healthcare facilities, particularly if a targeted population/clinical unit is selected, e.g. ICUs or neonatal wards. ${ }^{[21,22]}$ The feasibility and sensitivity of HAI detection using different surveillance strategies has not been evaluated in SA. We compared the performance of three HAI surveillance methods in an SA children's hospital using the CDC framework ${ }^{[23]}$ for evaluating public health surveillance programmes (reporting sensitivity, PPV, simplicity, flexibility, timeliness, acceptability and representativeness).

\section{Methods \\ Setting}

Tygerberg Children's Hospital (TCH) in Cape Town, SA, has 300 paediatric beds within a 1384 -bed academic hospital complex. Sick neonates, infants and children ( 0 - 14 years) are admitted to 13 neonatal and paediatric wards (including surgical wards, medical generalist wards, medical specialty wards, e.g. infectious diseases, haematology and oncology, and intensive care facilities); critically ill children requiring ventilation or inotropic support are managed in the 10-bed medical/surgical paediatric intensive care unit (PICU). There are approximately 17000 neonatal and paediatric admissions to TCH annually, with bed occupancy rates of $93 \%, 92 \%$ and $87 \%$ in the PICU and general and subspecialist wards, respectively, in 2015. The burden of infectious diseases is high, with HIV, tuberculosis, lower respiratory tract infections (RTIs) and gastroenteritis predominating. In 2013, the antenatal HIV prevalence in the Western Cape Province of SA was 19\% (v. 30\% nationally), and in 2012 the HIV prevalence in children ( 2 - 14 years) was $0.7 \%$ (v. $2.4 \%$ nationally).

\section{Investigation and management of HAIs at TCH}

Blood cultures are obtained from all children with suspected sepsis or severe infection in a focal site (e.g. pneumonia, cellulitis). Other laboratory samples are submitted at the discretion of attending clinicians, e.g. urine, pus or endotracheal aspirates. Empirical antibiotic therapy for HAIs usually includes meropenem, or ertapenem if Pseudomonas aeruginosa is considered unlikely and meningitis is excluded. Vancomycin is added if methicillin-resistant Staphylococcus aureus (MRSA) is a likely pathogen, e.g. suspected central-line sepsis or soft-tissue infection. Colistin, ciprofloxacin, fluconazole and amphotericin B are occasionally used empirically, although the hospital antimicrobial stewardship (AS) programme encourages use of narrower-spectrum, targeted therapy if culture results are available.

\section{Routine HAI surveillance at Tygerberg Hospital (including TCH)}

The Unit for Infection Prevention and Control (IPC) utilises laboratory surveillance of selected bacterial 'alert' pathogens from blood culture, urine, pus, endotracheal aspirates and sputum samples to calculate hospital-acquired bloodstream infection and other selected HAI rates. This targeted surveillance differs from the expanded laboratory surveillance method utilised in the present study, which included all pathogenic bacteria, fungi and selected viral pathogens. The only prospective clinical HAI surveillance at TCH has been conducted by neonatal ICU staff since 2013, reporting central line-associated bloodstream infection (CLABSI) rates (a type of device-associated HAI accounting for just $1.5 \%$ of paediatric HAI at our institution). ${ }^{[24]}$ Utilising laboratory surveillance data and individual patient referrals from clinicians, the IP practitioners conduct daily ward rounds to advise on transmission-based precautions, patient isolation, and other IPC-related management and staff education.

\section{Study design}

Prospective clinical surveillance (the reference method) was conducted in three paediatric wards (general paediatrics, paediatric surgery, infectious diseases/gastroenterology) and the PICU on weekdays from 1 May 2015 to 31 October 2015, documenting HAI events, microbiology/virology laboratory data and antimicrobial prescriptions among inpatients admitted for $\geq 48$ hours or transferred from another facility. Data on incident HAI events, laboratory data and antimicrobial prescriptions occurring over weekends were collected on the Monday ward rounds. CDC/NHSN definitions for $\mathrm{HAI}^{[14]}$ were used for both the reference method and the monthly HAI PPS. The CDC/NHSN defines an HAI in acute-care settings as 'a localized or systemic condition resulting from an adverse reaction to the presence of an infectious agent(s) or its toxin(s) that was not present or incubating' at the time of hospitalisation (bacterial colonisation and inflammation from noninfectious causes are excluded). HAI is confirmed when all elements of the CDC/NHSN criteria 'were first present together on or after the 3rd hospital day', and each HAI type has so-called 'site-specific criteria', e.g. SSI, skin and soft-tissue infection. 'Presumed' HAI infection events (not part of the CDC/NHSN classification) were defined as episodes of clinically diagnosed HAI without an identified source or positive laboratory tests, where broad-spectrum antimicrobials were initiated and continued for at least 5 calendar days. Each surveillance type was considered to have correctly identified an HAI event (identified by the reference method) if the HAI event was 'active' on the day of the PPS, any microbiology/virology investigation for suspected HAI yielded a pathogen, or any new prescription was boarded for meropenem, ertapenem, ciprofloxacin, vancomycin or colistin. The updated CDC guideline for public health surveillance programme evaluation ${ }^{[23]}$ was used to compare each surveillance method's performance for sensitivity, PPV, simplicity, flexibility, timeliness, acceptability and representativeness.

\section{Statistical analysis}

HAI events identified by the reference method were used to calculate HAI incidence (HAI events/1 000 patient days) and HAI prevalence (one or more HAI event/100 admissions). HAI incidence (in wards and the PICU) and overall HAI prevalence estimates obtained by the three comparator surveillance methods were calculated with 95\% confidence intervals (CIs). The sensitivity of each comparator surveillance method was calculated as number of HAI events detected/total events detected by the reference method. PPV was calculated as the proportion of 'HAI cases' detected by the comparator method that were confirmed by the reference method. Potential factors influencing HAI detection rates for each surveillance method were entered into a logistic regression model. A $p$-value of $<0.05$ was considered statistically significant. Stata statistical software version 13.0 (StataCorp, USA) was used.

\section{Ethical approval}

Research approval and waiver of individual informed consent were obtained from the Human Health Research Ethics Committee of 
Stellenbosch University (ref. no. S13/09/171), and institutional approval was obtained from Tygerberg Hospital (no reference number).

\section{Results}

During the 6-month surveillance period, 1347 children were transferred in and/or admitted for $\geq 48$ hours to the three wards and the PICU, generating 13401 patient days. The reference method detected 417 HAI events during 324 patient admission episodes for 296 discrete patients (1.4 HAI events per affected patient). The overall HAI incidence rate was high at $31 / 1000$ patient days $(95 \%$ CI 28.2 - 34.2), with a period prevalence of $22 / 100$ admissions ( $95 \%$ CI 28.2 - 34.2) (Table 1). HAI rates were highest among children admitted to the PICU (94.4/1 000 patient days (95\% CI 80.6 - 109.8)).

Sensitivity of alternative HAI surveillance methods was highest for antimicrobial prescriptions at $66.4 \%$ (95\% CI 61.8 - 70.8), followed by laboratory surveillance at $48.4 \%$ (95\% CI 43.7 - 53.2) (Table 1). Repeated PPSs were significantly less sensitive than antimicrobial and laboratory surveillance (24.9 (95\% CI 21.0 - 29.3); $p<0.001$ ). Combining antimicrobial and laboratory surveillance improved sensitivity to $84.7 \%$ (95\% CI 80.9 - 87.8).

PPVs were $100 \%$ for PPS, $55.2 \%$ (95\% CI $50.1-60.2)$ for laboratory surveillance and $88.5 \%$ (95\% CI 84.5 - 91.6) for antimicrobial prescriptions. Reasons for 'misidentification' of the 164 false positives identified by laboratory surveillance included pathogens representing colonisation only $(n=21)$, communityacquired pathogens $(n=129)$, duplicate laboratory specimens $(n=5)$, and pathogens identified from more than one sample site, e.g. bloodstream and urine $(n=9)$. Few false positives were identified by antimicrobial prescription surveillance $(n=36)$, with resistant community-acquired infections and complicated intraabdominal infections as the main indications for carbapenem or ciprofloxacin use in these cases. Combining antimicrobial and laboratory surveillance substantially reduced false-positive HAI identification ( $n=11)$ and resulted in an improvement of the PPV to $97 \%$ (95\% CI 94.6 - 98.4).

The alternative surveillance methods performed variably for detecting the four main HAI types: hospital-acquired pneumonia (HAP) $(n=185)$, urinary tract infection (UTI) $(n=45)$, laboratoryconfirmed bloodstream infection (LC-BSI) $(n=41)$, and SSI $(n=20)$ (Fig. 1). Compared with the reference method, repeated PPS detected $<30 \%$ of the four main HAI types other than SSI, whereas laboratory surveillance achieved the highest proportional detection rates for UTI, SSI and LC-BSI. Antimicrobial prescriptions achieved the highest detection rates for HAP. Combining antimicrobial and laboratory surveillance substantially improved detection of SSI and HAP events, and to a lesser extent UTI and LC-BSI detection.

Table 2 summarises the pathogens associated with four main HAI types. Klebsiella pneumoniae $(33 / 68,48.6 \%)$ and S. aureus $(13 / 25,52.0 \%)$ were the leading Gram-negative and Gram-positive

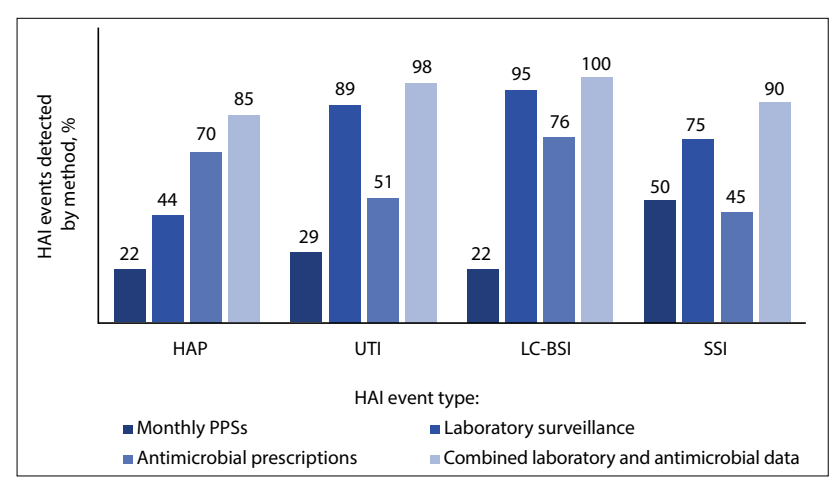

Fig. 1. Comparative HAI detection by surveillance method and HAI event type. The proportion of HAI events detected by alternative surveillance methods (when compared with the reference method's assumed HAI detection rate of $100 \%)$ is shown for four frequent HAI event types: HAP $(\mathrm{n}=185)$, UTI $(\mathrm{n}=45), L C-B S I(\mathrm{n}=41)$ and SSI $(\mathrm{n}=21)$.

Table 1. Comparative performance of HAI surveillance methods

\begin{tabular}{|c|c|c|c|c|c|}
\hline Measure & $\begin{array}{l}\text { Reference } \\
\text { method }\end{array}$ & PPSs & $\begin{array}{l}\text { Laboratory } \\
\text { surveillance }\end{array}$ & $\begin{array}{l}\text { Antimicrobial } \\
\text { prescriptions }\end{array}$ & $\begin{array}{l}\text { Combined } \\
\text { laboratory- } \\
\text { antimicrobial } \\
\text { surveillance }\end{array}$ \\
\hline HAI 'cases' detected by method, $n$ & 417 & 104 & 202 & 277 & 353 \\
\hline \multicolumn{6}{|l|}{ HAI incidence, /1 000 patient days ${ }^{*}(95 \%$ CI) } \\
\hline Overall (wards + PICU) & $\begin{array}{l}31.1 \\
(28.2-34.2)\end{array}$ & $\begin{array}{l}7.8 \\
(6.3-9.4)\end{array}$ & $\begin{array}{l}15.1 \\
(13.1-17.3)\end{array}$ & $\begin{array}{l}20.7 \\
(18.3-23.2)\end{array}$ & $\begin{array}{l}26.3 \\
(23.7-29.2)\end{array}$ \\
\hline PICU & $\begin{array}{l}94.4 \\
(80.6-109.8)\end{array}$ & $\begin{array}{l}20.5 \\
(14.1-28.7)\end{array}$ & $\begin{array}{l}56.5 \\
(45.7-68.9)\end{array}$ & $\begin{array}{l}72 \\
(59.9-85.8)\end{array}$ & $\begin{array}{l}88.8 \\
(75.4-103.8)\end{array}$ \\
\hline Paediatric wards & $\begin{array}{l}22.5 \\
(19.9-25.3)\end{array}$ & $\begin{array}{l}6 \\
(4.7-7.6)\end{array}$ & $\begin{array}{l}9.4 \\
(7.8-11.3)\end{array}$ & $\begin{array}{l}13.7 \\
(11.6-15.9)\end{array}$ & $\begin{array}{l}17.8 \\
(15.5-20.4)\end{array}$ \\
\hline HAI prevalence, ${ }^{\dagger} / 100$ admissions $(95 \% \mathrm{CI})$ & $\begin{array}{l}22 \\
(19.9-24.3)\end{array}$ & $\begin{array}{l}7.4 \\
(6.1-9)\end{array}$ & $\begin{array}{l}12 \\
(10.3-13.8)\end{array}$ & $\begin{array}{l}16.1 \\
(14.2-18.2)\end{array}$ & $\begin{array}{l}20.5 \\
(18.5-22.8)\end{array}$ \\
\hline Sensitivity, $\%$ (95\% CI) & $\begin{array}{l}\text { Reference } \\
\text { standard }\end{array}$ & $\begin{array}{l}24.9 \\
(21.0-29.3)\end{array}$ & $\begin{array}{l}48.4 \\
(43.7-53.2)\end{array}$ & $\begin{array}{l}66.4 \\
(61.8-70.8)\end{array}$ & $\begin{array}{l}84.7 \\
(80.9-87.8)\end{array}$ \\
\hline $\mathrm{PPV}, \$ \%(95 \% \mathrm{CI})$ & $\begin{array}{l}\text { Reference } \\
\text { standard }\end{array}$ & 100 & $\begin{array}{l}55.2 \\
(50.1-60.2)\end{array}$ & $\begin{array}{l}88.5 \\
(84.5-91.6)\end{array}$ & $\begin{array}{l}97 \\
(94.6-98.4)\end{array}$ \\
\hline \multicolumn{6}{|c|}{$\begin{array}{l}\text { *Patient days: sum of patients on each ward at } 08 \mathrm{~h} 00 \text { every day during the } 6 \text {-month study period }(13401 \text { patient days }=1610 \text { paediatric ICU }+11791 \text { three paediatric wards). } \\
\text { 'HAI prevalence: discrete patients with one or more HAI event } / 100 \text { patient admissions to the four selected wards (reference method }=296 / 1347 ; \mathrm{PPS}=100 / 1347 ; \text { laboratory surveillance }= \\
161 / 1347 ; \text { antimicrobial prescriptions }=217 / 1347 ; \text { combined laboratory }+ \text { antimicrobial data }=277 / 1346) \text {. } \\
\text { FSensitivity of the surveillance method: number of HAI events detected by the alternative method/total events detected by the reference method, e.g. HAI events detected by PPS/reference } \\
\text { method = } 104 / 417(24.9 \%) \text {. } \\
\text { 'PPV: the proportion of detected 'cases' who actually had an HAI event confirmed by the reference method, e.g. for antimicrobial prescriptions = true positives }(277) /[\text { true positives }(277)+\text { fals } \\
\text { positives (36)] }(88.5 \%) \text {. }\end{array}$} \\
\hline
\end{tabular}


Table 2. Pathogens associated with selected HAI types $(N=299)$

\begin{tabular}{|c|c|c|c|c|c|}
\hline Pathogen & $\begin{array}{l}\text { LC-BSI } \\
(N=41) \\
\end{array}$ & $\begin{array}{l}\text { CLABSI }^{*} \\
(N=7)\end{array}$ & $\begin{array}{l}\text { UTI } \\
(N=45)\end{array}$ & $\begin{array}{l}\text { SSI } \\
(N=21)\end{array}$ & $\begin{array}{l}\operatorname{HAP}^{\dagger} \\
(N=185)\end{array}$ \\
\hline \multicolumn{6}{|l|}{ Gram-negatives $(N=72)$} \\
\hline Klebsiella pneumoniae & 5 & 2 & 24 & 2 & 2 \\
\hline Enterobacter cloacae & 4 & 0 & 0 & 1 & 0 \\
\hline Escherichia coli & 5 & 0 & 7 & 4 & 0 \\
\hline Acinetobacter spp. & 3 & 0 & 0 & 0 & 0 \\
\hline Pseudomonas aeruginosa & 2 & 0 & 1 & 3 & 0 \\
\hline Serratia marcescens & 1 & 0 & 0 & 0 & 1 \\
\hline Salmonella non-typhi & 1 & 0 & 0 & 0 & 0 \\
\hline Morganella morganii & 0 & 0 & 0 & 2 & 0 \\
\hline Bordetella pertussis & 0 & 0 & 0 & 0 & 1 \\
\hline Stenotrophomonas maltophilia & 0 & 0 & 0 & 0 & 1 \\
\hline \multicolumn{6}{|l|}{ Gram-positives $(N=25)$} \\
\hline Staphylococcus aureus & 6 & 1 & 2 & 4 & 0 \\
\hline Enterococcus faecium & 3 & 0 & 1 & 0 & 0 \\
\hline Enterococcus faecalis & 1 & 0 & 1 & 0 & 0 \\
\hline $\begin{array}{l}\text { Coagulase-negative } \\
\text { staphylococci }\end{array}$ & 4 & 0 & 0 & 0 & 0 \\
\hline Leuconostoc spp. & 0 & 1 & 0 & 0 & 0 \\
\hline Streptococcus agalactiae & 1 & 0 & 0 & 0 & 0 \\
\hline \multicolumn{6}{|l|}{ Fungi $(N=18)$} \\
\hline Candida albicans & 3 & 1 & 6 & 0 & 0 \\
\hline C. glabrata & 0 & 2 & 1 & 0 & 0 \\
\hline C. parapsilosis & 2 & 1 & 0 & 0 & 0 \\
\hline C. lusitaniae & 0 & 0 & 2 & 0 & 0 \\
\hline \multicolumn{6}{|l|}{ Viruses $(N=93)$} \\
\hline Respiratory syncytial virus & - & - & - & - & 38 \\
\hline Adenovirus & - & - & - & - & 25 \\
\hline Parainfluenza virus & - & - & - & - & 14 \\
\hline Influenza virus & - & - & - & - & 5 \\
\hline Coronavirus OC43 & - & - & - & - & 4 \\
\hline Human metapneumovirus & - & - & - & - & 4 \\
\hline Rhinovirus & - & - & - & - & 2 \\
\hline Bocavirus & - & - & - & - & 1 \\
\hline No pathogen isolated & - & - & - & $3(14.2 \%)$ & $69(37.3 \%)$ \\
\hline No specimen sent & - & - & - & $2(9.6 \%)$ & $34(18.4 \%)$ \\
\hline
\end{tabular}

bacterial isolates, respectively, for LC-BSI, UTI and SSI events. Of the 61 Enterobacteriaceae isolated, 35 (57.4\%) were ESBL producers, and $3 / 13(23.1 \%)$ S. aureus isolates were MRSA. Viral pathogens (particularly respiratory syncytial virus (RSV) and adenovirus) predominated in HAP events, with $82 / 151$ patients (54.3\%) investigated yielding one or more RTI pathogens.

Factors associated with failure to detect an HAI (Table 2) were as follows: for PPSs, patient transfer (OR 2.0; 95\% CI 1.01 - 3.1) and a single HAI event (OR 2.8; 95\% CI 1.5 - 5.1); for laboratory surveillance, admission to a general ward (OR 2.3; 95\% CI 1.5 3.5 ) and age group 1 - 5 years (OR 2.1; 95\% CI 1.1 - 3.9), and for antimicrobial prescriptions, admission to a general ward (OR 1.8; $95 \%$ CI 1.1 - 2.9). Death was associated with significantly less chance of a 'missed' HAI event for the antimicrobial prescription surveillance method (OR 0.3; 95\% CI 0.1 - 0.9) (Table 3). Table 4 compares data on the qualitative elements of the CDC surveillance programme evaluation, and summarises the advantages, disadvantages and recommended settings for use of each surveillance method.

\section{Discussion}

The reference method measured HAI prevalence at $22 \%$, far exceeding rates of $4-5 \%$ reported in hospitalised children in high- 
Table 3. Factors associated with failure to detect $\mathrm{HAI}^{\star}$

\begin{tabular}{|c|c|c|c|c|c|c|c|c|c|c|c|c|}
\hline & \multicolumn{12}{|c|}{ Surveillance method } \\
\hline & \multicolumn{4}{|c|}{ Repeated PPSs } & \multicolumn{4}{|c|}{ Laboratory surveillance } & \multicolumn{4}{|c|}{ Antibiotic prescriptions } \\
\hline & $\begin{array}{l}\text { Uni- } \\
\text { variate } \\
\text { analysis } \\
\text { ( } p \text {-value) }\end{array}$ & $\begin{array}{l}\text { Multi- } \\
\text { variate } \\
\text { analysis } \\
\text { ( } p \text {-value) }\end{array}$ & $\begin{array}{l}\text { Odds } \\
\text { ratio }\end{array}$ & $95 \% \mathrm{CI}$ & $\begin{array}{l}\text { Uni- } \\
\text { variate } \\
\text { analysis } \\
\text { ( } p \text {-value) }\end{array}$ & $\begin{array}{l}\text { Multi- } \\
\text { variate } \\
\text { analysis } \\
\text { ( } p \text {-value) }\end{array}$ & $\begin{array}{l}\text { Odds } \\
\text { ratio }\end{array}$ & 95\% CI & $\begin{array}{l}\text { Uni- } \\
\text { variate } \\
\text { analysis } \\
\text { ( } p \text {-value) }\end{array}$ & $\begin{array}{l}\text { Multi- } \\
\text { variate } \\
\text { analysis } \\
\text { ( } p \text {-value) }\end{array}$ & $\begin{array}{l}\text { Odds } \\
\text { ratio }\end{array}$ & 95\% CI \\
\hline $\begin{array}{l}\text { Ward } \\
\text { type }\end{array}$ & 0.248 & - & - & - & $<0.001$ & $<0.001$ & 2.28 & $\begin{array}{l}1.48- \\
3.51\end{array}$ & 0.001 & 0.017 & 1.81 & $\begin{array}{l}1.11- \\
2.97\end{array}$ \\
\hline $\begin{array}{l}\text { Length of } \\
\text { stay }\end{array}$ & 0.023 & 0.24 & 0.99 & $\begin{array}{l}0.97- \\
0.99\end{array}$ & 0.90 & - & - & - & 0.016 & 0.27 & 0.99 & $\begin{array}{l}0.98- \\
1.00\end{array}$ \\
\hline $\begin{array}{l}\text { Outcome: } \\
\text { Death }\end{array}$ & $<0.001$ & 0.06 & 1.87 & $\begin{array}{l}0.95- \\
8.8\end{array}$ & 0.147 & - & - & - & 0.002 & 0.03 & 0.36 & $\begin{array}{l}0.14- \\
0.93\end{array}$ \\
\hline $\begin{array}{l}\text { Outcome: } \\
\text { Transfer }\end{array}$ & $<0.001$ & 0.04 & 2.0 & $\begin{array}{l}1.01- \\
3.07\end{array}$ & 0.147 & - & - & - & 0.002 & 0.30 & 0.77 & $\begin{array}{l}0.47- \\
1.26\end{array}$ \\
\hline $\begin{array}{l}\text { Single } \\
\text { HAI } \\
\text { event }\end{array}$ & 0.001 & 0.001 & 2.8 & $\begin{array}{l}1.53- \\
5.09\end{array}$ & 0.074 & - & - & - & 0.192 & - & - & - \\
\hline $\begin{array}{l}\text { Patient } \\
\text { risk } \\
\text { factor/s } \\
\text { for HAI }\end{array}$ & 0.987 & - & - & - & 0.321 & - & - & - & 0.803 & - & - & - \\
\hline $\begin{array}{l}\text { HIV } \\
\text { status }\end{array}$ & 0.419 & - & - & - & 0.72 & - & - & - & 0.241 & - & - & - \\
\hline $\begin{array}{l}\text { Age } \\
\text { category: } \\
1-5 \\
\text { years }\end{array}$ & 0.654 & - & - & - & 0.002 & 0.03 & 2.07 & $\begin{array}{l}1.08- \\
3.98\end{array}$ & 0.619 & - & - & - \\
\hline
\end{tabular}

income settings. ${ }^{[24,25]}$ Our study prevalence (including all HAI types) is similar (16.5\%) to that established for four HAI types (SSI, UTI, LC-BSI and RTIs) in a paediatric ward in Gauteng, SA, in 2005. ${ }^{[9]}$ Given the extreme paucity of data ${ }^{[1,26]}$ on paediatric HAI in Africa, we could not benchmark our HAI prevalence against other inpatient settings. The high HAI rate in our ICU (v. paediatric wards) is in keeping with published data for both LMICs and high-income countries (albeit three- to four-fold higher than their published HAI rates). ${ }^{[1]}$ Prevalence and incidence estimates produced by the combined laboratory-antimicrobial surveillance method were not significantly different (overlapping CIs) from those measured by the reference method, suggesting that combined laboratoryantimicrobial surveillance provides the most accurate approximation of the true HAI burden in our setting.

Repeated PPSs showed poorer sensitivity than laboratory and antimicrobial prescription surveillance. Monthly PPSs would have missed many infection events, including HAI-attributable mortality. This limitation of the PPS methodology can be partially mitigated by using period prevalence (infections occurring during a predefined period before the survey day) rather than point prevalence (which only includes HAIs present on the survey day). Zingg et al. ${ }^{[27]}$ established a $32 \%$ greater HAI yield when using period prevalence (HAI within 7 days of the survey date), with the additional cases attributed largely to identification of short-duration HAIs, e.g. lower RTIs and UTIs. This phenomenon may partly explain why our monthly PPSs performed better at detecting SSI events than other HAIs, as SSIs prolonged hospitalisation disproportionately to other HAI types, increasing the likelihood of being surveyed. We also identified that transfer out was associated with failure to detect HAI on PPS, reflecting the lower likelihood of a patient with HAI being present on a PPS day if they had been transferred out to complete antibiotic therapy at another facility.

Another limitation of PPSs is the inability to quantify the influence of seasonal fluctuations in community-acquired viral infections with potential for nosocomial spread. We documented very few healthcare-associated gastroenteritis events, possibly owing to the surveillance period (May - October are low-prevalence months for rotaviral disease in SA). Conversely, HAP events may have been overrepresented in our cohort, as the surveillance months included the peak winter hospitalisations for community-acquired RTIs. ${ }^{[28]}$

The perfect performance of PPSs at identifying true HAI is probably due to use of the same HAI case definitions and data collection tool as the reference method. The PPV achieved by antimicrobial prescription surveillance was similarly high, indicating that ultra-broad-spectrum antibiotics, e.g. carbapenems, were reserved mostly for HAIs. This finding can be ascribed in part to institutional AS interventions targeting clinicians, e.g. antimicrobial restriction policies and weekly AS ward rounds. The PPV of laboratory surveillance was poor, mainly because of difficulty in excluding community-acquired and colonising pathogens in 
Table 4. Comparison of HAI surveillance methods

\begin{tabular}{|c|c|c|c|c|}
\hline & Reference method ${ }^{\star}$ & PPSs & Laboratory surveillance & $\begin{array}{l}\text { Antimicrobial } \\
\text { prescriptions }\end{array}$ \\
\hline Simplicity $^{\dagger}$ & Most complex & Moderately complex & Less complex & Least complex \\
\hline Activities & $\begin{array}{l}\text { Daily ward rounds, data } \\
\text { capture + validation }\end{array}$ & $\begin{array}{l}\text { Monthly ward rounds, data } \\
\text { capture + validation }\end{array}$ & $\begin{array}{l}\text { Laboratory data and } \\
\text { hospital admissions, data } \\
\text { extraction + validation }\end{array}$ & $\begin{array}{l}\text { Pharmacy data and } \\
\text { hospital admissions, } \\
\text { data extraction }+ \\
\text { validation }\end{array}$ \\
\hline Resources & $\begin{array}{l}\text { IP data collector, data } \\
\text { capturer, hospital IT }\end{array}$ & $\begin{array}{l}\text { IP data collector, data } \\
\text { capturer, hospital IT }\end{array}$ & $\begin{array}{l}\text { Laboratory database/s, } \\
\text { hospital IT }\end{array}$ & $\begin{array}{l}\text { Pharmacy database, } \\
\text { hospital IT }\end{array}$ \\
\hline Time (hours/month) & 120 & 30 & 10 & 5 \\
\hline Flexibility ${ }^{\S}$ & Moderately flexible & Moderately flexible & Moderately flexible & Very flexible \\
\hline Timeliness & High & Moderate & $\begin{array}{l}\text { Low (unless using real- } \\
\text { time surveillance) }\end{array}$ & Low \\
\hline Acceptability" & Low & Moderate & Moderate to high & Moderate to high \\
\hline Representativeness ${ }^{* *}$ & High & Moderate & $\begin{array}{l}\text { Moderate to low } \\
\text { (influenced by frequency } \\
\text { and quality of laboratory } \\
\text { sampling) }\end{array}$ & $\begin{array}{l}\text { Moderate to low } \\
\text { (influenced by } \\
\text { availability of and } \\
\text { ability to extract } \\
\text { additional clinical data) }\end{array}$ \\
\hline Advantages & $\begin{array}{l}\text { Detects more HAI events } \\
\text { than other methods; } \\
\text { collects clinical data } \\
\text { simultaneously; establishes } \\
\text { whether infection is HA } \\
\text { v. HCA }\end{array}$ & $\begin{array}{l}\text { Less labour intensive; if } \\
\text { repeated regularly may } \\
\text { be helpful in establishing } \\
\text { trends; collects clinical data } \\
\text { simultaneously }\end{array}$ & $\begin{array}{l}\text { Less labour intensive; } \\
\text { additional data on } \\
\text { pathogen profile and } \\
\text { antibiotic susceptibility } \\
\text { patterns of the institution }\end{array}$ & $\begin{array}{l}\text { Least labour intensive; } \\
\text { additional data on } \\
\text { antibiotic consumption } \\
\text { patterns for HAI at the } \\
\text { institution; can identify } \\
\text { HAI events even if rates } \\
\text { of laboratory sampling } \\
\text { or pathogen yields are } \\
\text { low }\end{array}$ \\
\hline Disadvantages & Labour intensive & $\begin{array}{l}\text { Misses many HAI events; } \\
\text { detection rate could be } \\
\text { improved if include all HAI } \\
\text { events occurring within } 7 \\
\text { days preceding the } \text { PPS }^{[26]}\end{array}$ & $\begin{array}{l}\text { Sensitivity depends on } \\
\text { frequency and quality } \\
\text { of laboratory samples } \\
\text { collected; difficult to } \\
\text { distinguish colonisation } \\
\text { from infection; cannot } \\
\text { distinguish HA from HCA } \\
\text { infection events }\end{array}$ & $\begin{array}{l}\text { Additional data on HAI } \\
\text { type and indication for } \\
\text { antibiotic and patient } \\
\text { demographics may not } \\
\text { be available }\end{array}$ \\
\hline Suitable contexts & $\begin{array}{l}\text { Hospital settings } \\
\text { with experienced IP } \\
\text { practitioners, IT analyst + } \\
\text { epidemiology support }\end{array}$ & $\begin{array}{l}\text { Hospital settings } \\
\text { with experienced IP } \\
\text { practitioner/s + some IT } \\
\text { analyst support }\end{array}$ & $\begin{array}{l}\text { Hospitals with high use } \\
\text { of laboratory tests for } \\
\text { investigation of HAI and } \\
\text { available IT/data analyst } \\
\text { assistance }\end{array}$ & $\begin{array}{l}\text { Hospitals with } \\
\text { insufficient resources } \\
\text { to perform other } \\
\text { surveillance, moderate } \\
\text { IT support/searchable } \\
\text { pharmacy database }\end{array}$ \\
\hline \multicolumn{5}{|c|}{$\begin{array}{l}\text { FTime calculations were based on surveillance conducted in } 1 \text { PICU (10 beds) and } 3 \text { paediatric wards (bed complement }=83 \text { ). } \\
\text { 'Simplicity includes the surveillance method structure, method of data collection and analysis, resources needed and ease of operation. } \\
\text { sFlexibility includes the system's ability to adapt to changing information or operating conditions with minimal changes to time, personnel or operational costs, e.g. changes to HAI case } \\
\text { definitions and use of standard data formats allowing easy integration with other systems. } \\
\text { 'Timeliness is the time interval between the onset of the HAI event and its reporting to those in charge of prevention and control efforts. }\end{array}$} \\
\hline
\end{tabular}

the absence of clinical information. Combining antimicrobial and laboratory surveillance achieved a very high PPV, suggesting that it is an efficient way to monitor HAI events (with few false positives that would need folder review to exclude from HAI estimates).

Laboratory surveillance performed well for three of the four main HAI types (LC-BSI, SSI and UTI), but failed to detect over half of HAP events. However, combined laboratory-antimicrobial surveillance resulted in improved detection of all main HAI types (particularly SSI and HAP events, which were poorly detected by individual surveillance methods). The poor sensitivity of laboratory surveillance for HAP may be due to low rates of laboratory testing for viral pathogens with polymerase chain reaction (PCR) outside the PICU and failure to detect bacterial respiratory pathogens with standard laboratory investigations. 
The influence of viral pathogens on paediatric HAI rates is probably grossly underestimated. In a 3-month study of hospitalised children in Soweto, 15/130 (11.6\%) clinically diagnosed with 'nosocomial sepsis' had confirmed nosocomial RSV infection. ${ }^{[2]}$ At Red Cross War Memorial Children's Hospital in Cape Town, 22/226 RSV infections $(9.5 \%)$ were nosocomially acquired in $2012 .{ }^{[30]}$

It is notable that laboratory surveillance (our institution's current surveillance method) was less sensitive than antibiotic prescription and combined laboratory-antimicrobial surveillance. This finding has implications for institutions with low rates of laboratory investigation of suspected HAI events; in such settings, laboratory surveillance may miss an even greater proportion of HAIs, which would still be detected by surveillance of antimicrobial prescriptions. In addition, laboratory surveillance was two times less likely to identify HAI in children aged 1 - 5 years, possibly because they undergo fewer laboratory investigations than infants who have nonspecific clinical presentation of sepsis necessitating more extensive diagnostic testing.

In keeping with previous SA studies, K. pneumoniae and $S$. aureus were the most frequently isolated HAI pathogens with a high prevalence of antimicrobial-resistant phenotypes. ${ }^{[28]}$ Viral pathogens were identified in over half of all patients with HAP who underwent laboratory testing, highlighting the importance of laboratory identification of pathogens in children with RTI (who serve as reservoirs of nosocomial virus transmission). In $18.4 \%$ of HAP events, no respiratory pathogen testing was performed, representing missed opportunities for identification and isolation of patients with transmissible pathogens.

The decision on which antimicrobials to include in prescriptions surveillance should be determined by the institution's empirical antimicrobial recommendations for HAI. The degree to which these antimicrobials are reserved for HAI treatment and the extent of resistant community-acquired infections will influence the HAI detection rate, potentially generating false-positive HAI events. Conversely, a prescription of antimicrobial/s other than the institution's empirical HAI therapy agents may result in 'missed' HAI events or false negatives.

We identified that hospitalisation in a ward (as opposed to the PICU) doubled the odds of failure to detect HAI (for both laboratory and antimicrobial surveillance methods). This finding is explained by the less frequent use of and lower yield of laboratory investigations in the wards as well as lower carbapenem utilisation rates. Death was associated with significantly less chance of 'missing' an HAI event by antimicrobial prescription surveillance, possibly because most critically ill children hospitalised for $>48$ hours would be prescribed a carbapenem antibiotic empirically.

Given the failure of alternative methods to achieve high HAI detection rates, combining surveillance methods may achieve superior sensitivity (as demonstrated by combination of our antimicrobial and laboratory data to produce test sensitivity of $85 \%$ ). Another possible strategy would be use of antimicrobial prescriptions to monitor certain HAI types with low laboratory testing rates and pathogen yield (e.g. HAP) and to use laboratory surveillance for HAI events with higher pathogen isolation rates (e.g. UTI, LC-BSI and SSI). This would, however, require the pharmacy to record clinical indications for HAI therapy (which was recently implemented at some institutions through a dedicated antimicrobial prescription chart designed by the South African Antimicrobial Stewardship Programme). ${ }^{[31]}$

Furthermore, increased use of appropriate laboratory tests for viral pathogens in cases of presumed HAI should be encouraged to assist with AS efforts. This strategy would avoid empirical antibiotic treatment of presumed HAI based only on clinical suspicion, and limit antibiotic duration in cases where viral pathogens are identified or presumed HAI events where no pathogens are identified, after appropriate laboratory investigations.

Repeated PPSs should not be discounted in low-resource settings, although in our cohort this method had the lowest sensitivity and required the greatest resources (time and labour). A distinct advantage of the PPS methodology is that a standardised, validated data collection tool can be used regionally or nationally, reducing inter- and intra-observer variability and facilitating benchmarking of individual facilities. Greater inter-institution variability in HAI rates would be expected using laboratory and/or antimicrobial prescription surveillance owing to variable specimen collection practices, laboratory methods and prescribing practices. Another advantage of PPSs is the possibility of collecting patient demographic data to identify patients at highest risk of HAI, allowing for targeting of HAI prevention interventions.

Using the CDC guidelines for evaluating surveillance systems, we identified wide variability in performance attributes of each surveillance method. Although the resources required to implement the reference surveillance method at our institution are not currently available, prospective, continuous HAI surveillance would provide timely (in real time) and representative ('whole-house') data. Laboratory and antimicrobial prescription surveillance methods appear the most attractive, most flexible and least resource-intensive options, but would provide less frequent HAI reports (weekly or monthly) and less complete data (not every case of HAI will be detected) in our context. Real-time laboratory data surveillance (if combined with additional clinical data collection) could, however, provide more accurate, timely and representative data.

Limitations of our study include the single study site, selection of four 'representative' wards, with exclusion of neonatal wards and the neonatal ICU, the study period sampled (May - October), which may have underestimated seasonal fluctuations in HAI (from nosocomial propagation of community-acquired viral infections), the use of antibiotic prescription data from patient records rather than from pharmacy databases, and microbiology/virology laboratory testing at clinicians' discretion with possible underdiagnosis of HAI events by laboratory surveillance (although this could also be construed as a strength, in that it represents the performance of laboratory surveillance in a 'real-world', current-practice setting).

In LMICs where the burden of HAIs is greatest, many healthcare facilities are poorly equipped to conduct prospective clinical HAI surveillance. Consequently, published data on the epidemiology of HAIs in developing countries are scant, but could be increased through use of simpler, less resource-intensive surveillance methods than the reference standard of prospective, clinical HAI surveillance. No single method will approximate the reference method or perform uniformly in all settings. Individualisation of HAI surveillance recommendations is therefore needed, considering available resources and the practice context. Nevertheless, our study suggests that laboratory surveillance, antimicrobial prescriptions, PPSs or combinations thereof are feasible alternatives to conventional clinical HAI surveillance.

\section{Conclusions}

SA paediatric wards should select an HAI surveillance method based on available resources, expertise and technology infrastructure. Where clinical HAI surveillance is not possible, monitoring of antimicrobial prescriptions in combination with laboratory data analysis appears a reasonable alternative. 
Author contributions. All the authors contributed to study design and critical review of the manuscript. AD carried out the data collection, data cleaning and statistical analysis (with assistance from Ms Tonya Esterhuizen of the Stellenbosch University Biostatistics Unit, Centre for Evidence-based Healthcare). All the authors read and approved the final manuscript.

Acknowledgements. The primary author (AD) is supported by the South African Medical Research Council's Clinician Researcher Programme and the Discovery Foundation Academic Fellowship, but these funding bodies played no role in the design, data collection, data analysis and interpretation, or writing of the article. The authors wish to thank the staff, patients and parents of TCH and Ms Tonya Esterhuizen.

1. Allegranzi B, Bagheri Nejad S, Combescure C, et al. Burden of endemic health-care-associated infection in developing countries: Systematic review and meta-analysis. Lancet 2011;377(9761):228241. http://dx.doi.org/10.1016/S0140-6736(10)61458-4

2. Edwards JR, Pollock DA, Kupronis BA, et al. Making use of electronic data: The National Healthcare Safety Network eSurveillance Initiative. Am J Infect Control 2008;36(3 Suppl):S21-S26. http://dx.doi. org/10.1016/j.ajic.2007.07.007

3. Zarb P, Coignard B, Griskeviciene J, et al. The European Centre for Disease Prevention and Control (ECDC) pilot point prevalence survey of healthere-associted infections and antimicrobs Euro Surveill 2012;17(46): article 2. http://wwwe eurosurveillance 0 g/ViewArticle aspx? ArticleId=20316 (accessed 5 December 2016)

4. Gastmeier P, Behnke M, Breier AC, et al. [Healthcare-associated infection rates: measuring and comparing. Experiences from the German National Nosocomial Infection Surveillance System (KISS) and from other surveillance systems]. Bundesgesundheitsblatt Gesundheitsforschung Gesundheitsschutz 2012;55(11-12):1363-1369. http://dx.doi.org/10.1007/s00103-012-1551-y

5. Reilly J, Cairns S, Fleming S, et al. Results from the second Scottish national prevalence survey: The changing epidemiology of healthcare-associated infection in Scotland. J Hosp Infect 2012;82(3):170 174. http://dx.doi.org/10.1016/j.jhin.2012.07.024

6. Rothe C, Schlaich C, Thompson S. Healthcare-associated infections in sub-Saharan Africa. J Hosp Infect 2013;85(4):257-267. http://dx.doi.org 10.1016/j.jhin.2013.09.008

7. Duse AG. Infection control in developing countries with particular emphasis on South Africa. South Afr J Epidemiol Infect 2005;20(2):37-41. http://www.tandfonline.com/doi/pdf/10.1080/10158782.2005.1 1441230 (accessed 5 December 2016).

8. Visser A, Moore DP, Whitelaw A, et al. Global antibiotic resistance partnership situation analysis: Antibiotic use and resistance in South Africa: Part VII. Interventions. S Afr Med J 2011;101(8):587595

9. Duse AG. Surveillance of healthcare-associated infections (HCAIs) South Africa. http://www.cddep. Duse AG. Surveillance of healthcare-associated infections (HCAIs) So
org/sites/default/files/prof_adriano_duse-2_0.pdf (accessed 1 May 2016).

10. Durlach R, Mcllvenny G, Newcombe RG, et al. Prevalence survey of healthcare-associated infection in Argentina: Comparison with England, Wales, Northern Ireland and South Africa. J Hosp Infect 2012;80(3):217-223. http://dx.doi.org/10.1016/j.jhin.2011.12.001

11. National Department of Health, South Africa. National Core Standards for Health Establishments in South Africa. Pretoria: NDoH, 2011. http://www.rhap.org.za/wp-content/uploads/2014/05/NationalCore-Standards-2011-1.pdf (accessed 3 May 2016).

12. Horan TC, Gaynes RP. Surveillance of nosocomial infections. In: Mayhall CG, ed. Hospital Epidemiology and Infection Control. 3rd ed. Philadelphia: Williams \& Wilkins, 2004:1659-1702

13. Talbot TR, Bratzler DW, Carrico RM, et al. Public reporting of health care-associated surveillance data Recommendations from the Healthcare Infection Control Practices Advisory Committee. Ann Intern Med 2013;159(9):631-635. http://dx.doi.org/10.7326/0003-4819-159-9-201311050-00011
14. Centers for Disease Control. CDC/NHSN surveillance definition of healthcare-associated infection and criteria for specific types of infections in the acute care setting. 2016. http://www.cdc.gov/nhsn/ (DFs/pscMar

15. Klompas M, Yokoe DS. Automated surveillance of health care-associated infections. Clin Infect Dis 2009;48(9):1268-1275. http://dx.doi.org/10.1086/597591

16. Stevenson KB, Khan Y, Dickman J, et al. Administrative coding data, compared with CDC/NHSN criteria, are poor indicators of health care-associated infections. Am J Infect Control 2008;36(3):155164. http://dx.doi.org/10.1016/j.jaic.2008.01.004

17. Julian KG, Brumbach AM, Chicora MK, et al. First year of mandatory reporting of healthcareassociated infections, Pennsylvania: An infection control-chart abstractor collaboration. Infect Control Hosp Epidemiol 2006;27(9):926-930. http://dx.doi.org/10.1086/507281

18. Sherman $\mathrm{ER}$, Heydon $\mathrm{KH}, \mathrm{St}$ John $\mathrm{KH}$, et al. Administrative data fail to accurately identify cases of healthcare-associated infection. Infect Control Hosp Epidemiol 2006;27(4):332-337. http://dx.doi, org/10.1086/502684

19. Pokorny L, Rovira A, Martin-Baranera M, Gimeno C, Alonso-Tarres C, Vilarasau I. Automatic detection of patients with nosocomial infection by a computer-based surveillance system: A validation study in a general hospital. Infect Control Hosp Epidemiol 2006;27(5):500-503. http://dx.doi. org/10.1086/502685

20. Lowman W. Active surveillance of hospital-acquired infections in South Africa: Implementation, impact and challenges. S Afr Med J 2016;106(5):489-493. http://dx.doi.org/10.7196/SAMT.2016. v106i5.10783

21. Sarvikivi E, Kärki T, Lyytikäinen O; Finnish NICU Prevalence Study Group. Repeated prevalence surveys of healthcare-associated infections in Finnish neonatal intensive care units. J Hosp Infect 2010;76(2):156-160. http://dx.doi.org/10.1016/j.jhin.2010.03.020

22. Stoesser N, Emary K, Soklin S, et al. The value of intermittent point-prevalence surveys of healthcareassociated infections for evaluating infection control interventions at Angkor Hospital for Children, Siem Reap, Cambodia. Trans R Soc Trop Med Hyg 2013;107(4):248-253. http://dx.doi.org/10.1093/ trstmh/trt005

23. German RR, Lee LM, Horan JM, Milstein RL, Pertowski CA, Waller MN; Guidelines Working Group Centers for Disease Control and Prevention (CDC). Updated guidelines for evaluating public health surveillance systems: Recommendations from the Guidelines Working Group. MMWR Recomm Rep 2001;50(RR-13):1-35.

24. Magill SS, Edwards JR, Bamberg W, et al.; Emerging Infections Program Healthcare-Associated Infections and Antimicrobial Use Prevalence Survey Team. Multistate point-prevalence survey of health care-associated infections. N Engl J Med 2014;370(13):1198-1208. http://dx.doi.org/10.1056/ NEJMoa1306801

25. European Centre for Disease Prevention and Control (ECDC). Point Prevalence Survey of Healthcareassociated Infections and Antimicrobial Use in European Acute Care Hospitals. Stockholm: ECDC, 2013. http://ecdc.europa.eu/en/publications/publications/healthcare-associated-infections-antimicrobialuse-pps.pdf (accessed 30 November 2016)

26. Nejad SB, Allegranzi B, Syed SB, Ellis B, Pittet D. Health-care-associated infection in Africa: A systematic review. Bull World Health Organ 2011;89(10):757-765. http://dx.doi.org/10.2471/ BLT.11.088179

27. Zingg W, Huttner BD, Sax H, Pittet D. Assessing the burden of healthcare-associated infections through prevalence studies: What is the best method? Infect Control Hosp Epidemiol 2014;35(6):674684. http://dx.doi.org/10.1086/67642

28. Dramowski A, Whitelaw A, Cotton MF. Burden, spectrum and impact of healthcare-associated infection at a South African children's hospital. J Hosp Infect 2016;94(4):364-372. http://dx.doi. rg/10.1016/j.jhin.2016.08.022

29. Madhi SA, Ismail K, O'Reilly C, Cutland C. Importance of nosocomial respiratory syncytial virus infections in an African setting. Trop Med Int Health 2004;9(4):491-498. http://dx.doi.org/ $10.1111 / \mathrm{j} .1365-3156.2004 .01221 . \mathrm{x}$

30. Oladokun R, Muloiwa R, Hsiao NY, Valley-Omar Z, Nuttall J, Eley B. Clinical characterisation and phylogeny of respiratory syncytial virus infection in hospitalised children at Red Cross War Memorial Children's Hospital, Cape Town. BMC Infect Dis 2016;16:236. http://dx.doi.org/10.1186/s12879-016$1572-5$

31. Mendelson M. Role of antibiotic stewardship in extending the age of modern medicine. S Afr Med J 2015;105(5):414-418. http://dx.doi.org/10.7196/SAMJ.9635

Accepted 3 November 2016 